\title{
Directed Self-Assembly of Lipid Nanotubes from Inverted Hexagonal Structures
}

\author{
Kaori Sugihara, ${ }^{\dagger, *}{ }^{*}$ Mohamed Chami, ${ }^{\ddagger}$ Imre Derényi, ${ }^{\S}$ János Vörös, ${ }^{\dagger}$ and Tomaso Zambelli ${ }^{\dagger}$ \\ ${ }^{\dagger}$ Laboratory of Biosensors and Bioelectronics, Institute for Biomedical Engineering, ETH Zurich, Gloriastrasse 35, CH-8092 Zurich, Switzerland, ${ }^{\ddagger} \mathrm{C}-\mathrm{CINA}$, Center of Cellular \\ Imaging and Nano-analytics, Biozentrum, University of Basel, 26 Mattenstrasse, Basel, Switzerland, and ${ }^{\S}$ Department of Biological Physics, Eotvos University, \\ Pazmany P. stny. 1A, H-1117 Budapest, Hungary, and ELTE-MTA "Lendulet" Biophysics Research Group, Pazmany P. stny. 1A, H-1117 Budapest, Hungary
}

$1 \mathrm{D}$ lipid structures (e.g., helical structures, hollow cylindrical tubules) self-assemble in solution, induced by the lipid phase transition. ${ }^{1-9}$ Metallization of such $1 \mathrm{D}$ lipid structures results in quick and cheap metal nanotubes, ${ }^{1}$ although their patterning on surfaces requires another effort ${ }^{10-12}$ such as manual manipulation. ${ }^{13}$ The self-assembly of lipids is also interesting from a biological perspective because it has been linked to the origin of life. ${ }^{14} 1 \mathrm{D}$ lipid structures have been also shown to transport intercellular organelles ${ }^{15,16}$ and transmit electrical signals ${ }^{17}$ between eukaryotic cells and between bacteria.

On the other hand, one can manually form lipid micro- to submicrotubules by pulling a part of vesicles or cell membranes with a pipet, ${ }^{18-22}$ a microneedle, ${ }^{23}$ or optical tweezers. ${ }^{17}$ Microfluidic systems have been also used to fabricate similar structures by extruding lipid membranes through a microaperture, ${ }^{24}$ or by controlling the lipid hydration, ${ }^{25}$ shear force, ${ }^{26}$ and the aqueous entrapment within flow cells. ${ }^{27}$ In such works, the lipid-tubule formation is fundamentally based on the shape transformation of lamellar structures by applying a point load.

1,2-Dioleoyl-sn-glycero-3-phosphoethanolamine (DOPE) is a zwitterionic conicalshaped lipid. It has garnered attention due to its inverted-hexagonal phase $\left(\mathrm{H}_{\|}\right)$at full hydration and room temperature (Figure 1a). ${ }^{28-30}$ In aqueous solution, they form $\mathrm{H}_{\|}$blocks, frequently characterized by cryo-electron microscopy. ${ }^{31,32}$ The cytoplasmic membrane of Escherichia coli consists of $80 \%$ PE-based lipids, ${ }^{33}$ such as DOPE used in this work. The reason for the extremely high concentration of PE-based lipids in the bacteria remains elusive, although the conical shape of the lipid is known to play a key role in membrane curvature and vesicle fusion. ${ }^{34,35}$

\section{ABSTRACT}
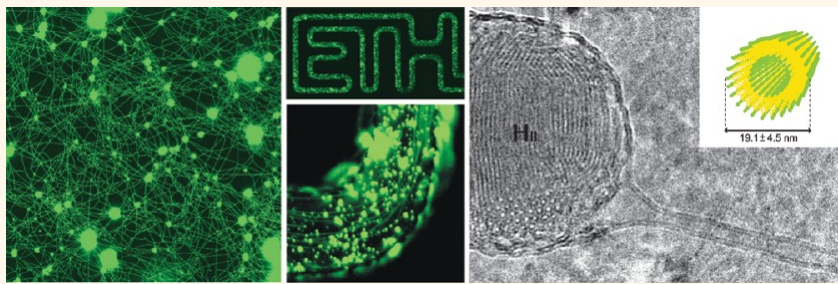

Conventional lipid-tube formation is based on either a tube phase of certain lipids or the shape transformation of lamellar structures by applying a point load. In the present study, lipid blocks in inverted hexagonal phase made of 1,2-dioleoyl-sn-glycero-3-phosphoethanolamine (DOPE) were shown to protrude lipid nanotubes upon a fluid-dynamic flow on polyelectrolytefunctionalized surfaces in physiological buffer solution. The outer diameter of the tubes is $19.1 \pm 4.5 \mathrm{~nm}$ and their lengths are up to several hundred micrometers. The method described enables the alignment and patterning of lipid nanotubes into various (including curvy) shapes with a microfluidic system.

KEYWORDS: 1,2-dioleoyl-sn-glycero-3-phosphoethanolamine (DOPE) · inverted hexagonal structure $\cdot$ directed self-assembly $\cdot$ lipid nanotubes · polyelectrolytes · microfluidic systems · cryo-electron microscopy

In this work, we report that the DOPE $\mathrm{H}_{\|}$ blocks adsorbed on a polyelectrolytefunctionalized surface transformed their shape into single-bilayer-wall tubes with an outer diameter of $19.1 \pm 4.5 \mathrm{~nm}$ and the length of nearly $1 \mathrm{~mm}$ upon the application of a solution flow. Distinct from other shear-force driven microtubule assemblies, (i) the diameter is a couple of $10 \mathrm{~nm}$, yielding the aspect ratio of $10^{5}$, and (ii) the growth mechanism involves the mixture of two phases; inverted hexagonal phase and lamellar phase. The main technical advantages of the process are that the assembly occurs directly on surfaces in the presence of flow and nanowire alignment is controlled by the flow direction. These characteristics allowed the lipid nanotubes to be patterned in a simple,

\section{* Address correspondence to sugihara@is.mpg.de.}

Received for review February 7, 2012 and accepted August 3, 2012.

Published online August 03, 2012 10.1021/nn300557s

() 2012 American Chemical Society 


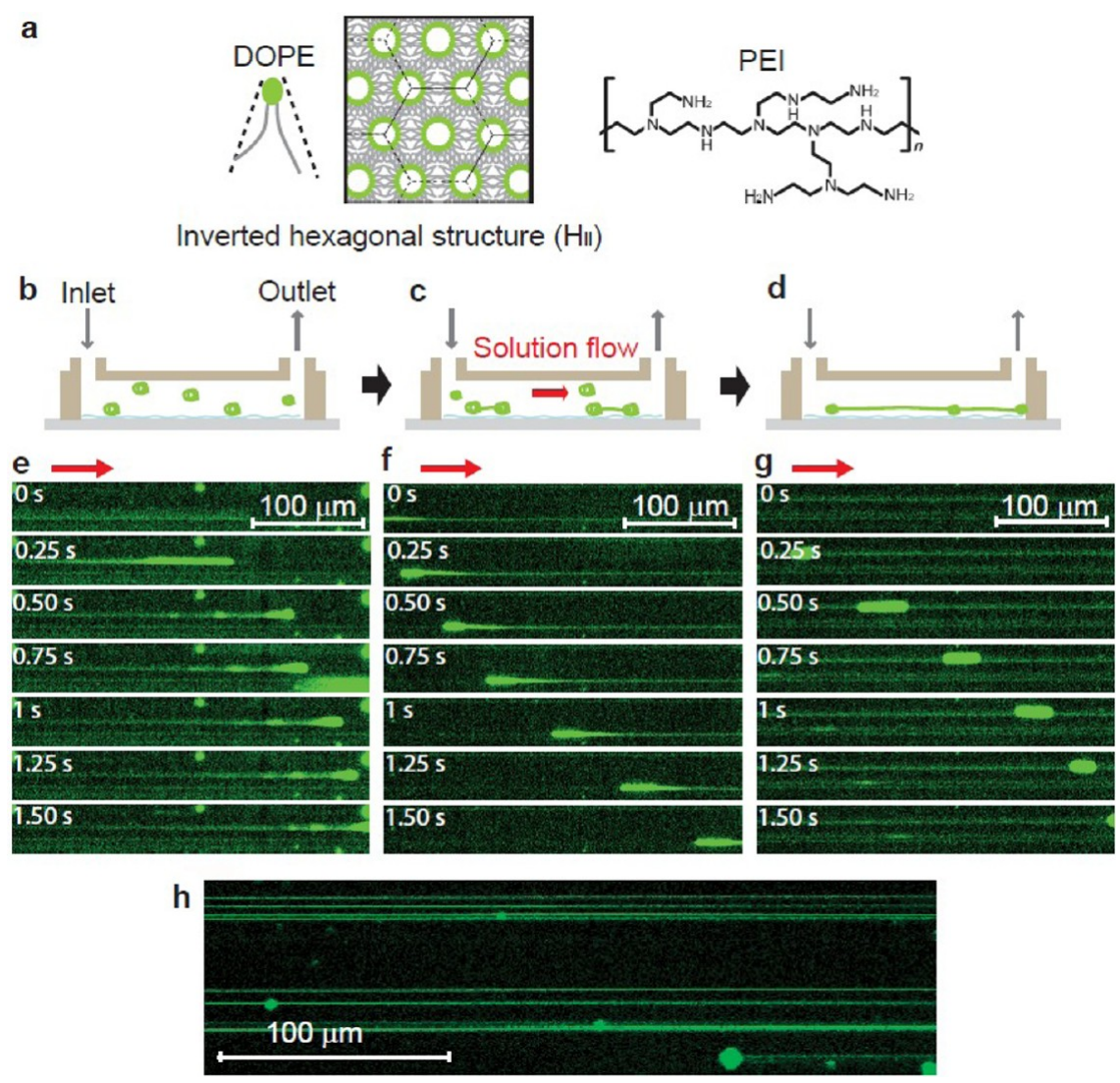

Figure 1. Directed self-assembly of lipid nanotubes. (a) Schematics of 1,2-dioleoyl-sn-glycero-3-phosphoethanolamine (DOPE) and PEI chemical structure. The $100 \%$ hydrated DOPE is in an inverted hexagonal phase $\left(H_{11}\right)$ in a physiological environment. The coating of a glass slide with polycation is possible because an oxygen-plasma-cleaned glass surface is negatively charged. (b-d) Schematics of the procedure of directed self-assembly of the lipid nanotubes, including (b) the adsorption of DOPE blocks on a PEI-coated glass slide, (c) rearrangement of the DOPE molecules directed by a shear stress, and (d) the formation of the lipid nanotubes. $(e-g)$ Three time series of snapshots from a movie during the assembly ( $0.25 \mathrm{~s} /$ frame). (e) Nanotube formation, (f) nanotube detachment, and (g) one-dimensional transport of a lipid block along a tube were observed. The red arrows indicate the direction of the flow. (h) A confocal laser scanning microscope (CLSM) image of formed lipid nanotubes.

straightforward, and high-throughput manner with microfluidic systems. In addition, lipids in the nanotubes exhibited a high mobility, which allowed the transport of lipid blocks along the wire. It is also intriguing to observe that a lipid that is so abundant in bacteria can form nanotubes, transports molecules along the nanotube, and disappears by itself in a physiological environment.

\section{RESULTS AND DISCUSSIONS}

To prepare the lipid solution, chloroform-stored DOPE was added to a flask, dried, resuspended with a physiological buffer solution, and sonicated. DOPE exists in the inverted-hexagonal phase $\left(\mathrm{H}_{\|}\right)$at full hydration, physiological $\mathrm{pH}, 150 \mathrm{mM} \mathrm{NaCl}$, and room temperature (Figure 1a). ${ }^{28-30}$ Upon sonication, lipids detach from the flask wall and form blocks of $\mathrm{H}_{\|}$phase. ${ }^{32} \mathrm{~A}$ glass slide was coated with a polycation, polyethyleneimine (PEI, Figure 1a). The DOPE blocks simply adsorbed onto the PEl-functionalized surface (Figure 1b). Upon a solution flow, the lipid blocks transform their shape, forming nanotubes (Figure $1 \mathrm{c}, \mathrm{d}$ ).
Representative snapshots of a movie taken during the directed self-assembly of the nanotubes are shown in Figure $1 \mathrm{e}-\mathrm{g}$ (part of the movie and other snapshots are shown in Supporting Information, movie S1 and Figure S1). The lipids showed three characteristic phenomena: (1) nanotube formation (Figure 1e), (2) nanotube detachment (Figure 1f), and (3) 1D transport of a lipid block on a tube (Figure 1g). Nanotube formation was initiated by the lipid block movement, with the nanotube assembling as a tail on the lipid block (Figure 1e). On the other hand, a created nanotube detached after a lipid block passed over it (Figure 1f). These two events indicate that the lipid block acts as a lipid reservoir, providing lipids to and collecting lipids from the nanotubes. In addition, Figure 1e shows that some lipid blocks remained without changing their location, indicating a distribution in the force required to move the blocks. One-dimensional transport of a lipid block along a tube was observed (Figure 1g), suggesting lipid mobility in the nanotubes. Assembled nanotubes were well-oriented along the flow and reached lengths of several hundred micrometers 

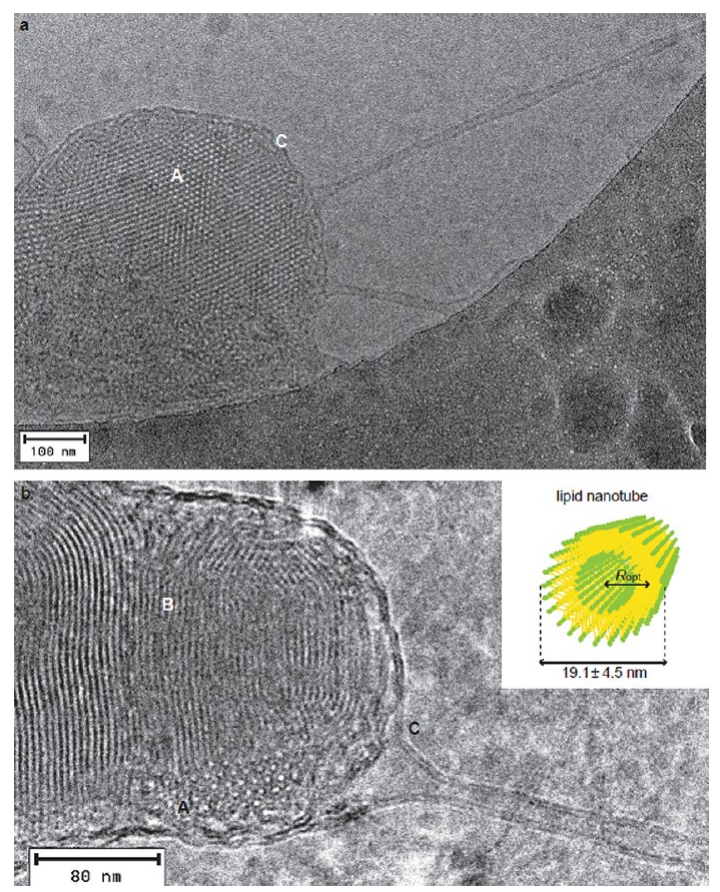

Figure 2. Cryo TEM images of the lipid nanotubes. (a) The hexagonal pattern typical for $\mathrm{H}_{\|}$is imaged in the lipid block. Single-bilayer-wall tubes are protruding from the block. (b) High-resolution image of the lipid block-tube junction. A schematic of the lipid nanotube is shown as an inset.

(Figure 1h). This length is among the longest reported for 1D lipid structures. ${ }^{1}$

Polyelectrolytes are charged polymers that weakly interact with electrically neutral lipids. ${ }^{36}$ The first key step in nanotube formation was the PEl-coating of the glass surface, which allowed the adsorbed blocks to maintain their block-structure. In addition to PEI, the polycation poly-L-lysine (PLL) induced a similar behavior of the lipid blocks together with nanotube formation (Supporting Information, Figure S2a). By contrast, adsorbed lipid blocks did not remain intact on a bare glass slide, but instead formed a mixture of lipid patches and ribbon-like structures (Supporting Information, Figure S2b). The second key step is the application of solution flow. This step induces nanotube formation as a tail on the block. From Figure 1e it can be deduced that the lipid block is attached locally to the surface, while the nonattached part of the lipid block can be displaced by the flow, disentangling the lipid block into a tube.

To study the internal structure of the tubes, samples were imaged by cryoTEM (Figure 2). The hexagonal pattern ( $A$ in Figure $2 \mathrm{ab}$ ) and the stripes that correspond to the side view ( $B$ in Figure $2 b$ ) typical for $\mathrm{H}_{\|}$ structures $^{37,38}$ were imaged for the lipid blocks as expected. The outermost layer of the block is a bilayer ( $C$ in Figure 2ab), which is connected to $H_{\|}$structure, being mediated by a loose lamellar structure in between. From the block, tubes are protruding. The highresolution image in Figure $2 \mathrm{~b}$ shows that the structure of the tube is a single-bilayer wall tube with an outer diameter of $19.1 \pm 4.5 \mathrm{~nm}$ (inset in Figure 2). These tubes in the images assembled directed by turbulent flows applied with a pipet in open flow cells (pipetting applies shear forces of a maximum of a few $\mathrm{N} / \mathrm{m}^{2}$ randomly, depending on the location). ${ }^{39}$ Regardless of the large variation in the force, the standard deviation of the tube diameter is surprisingly small. It implies that the tube diameter is independent of the applied force and it has an energetically preferable magnitude. The image of the lipid block-tube junction revealed that the tube is an expansion of the outermost bilayer (C in Figure 2b). Importantly, three structures (i.e., $\mathrm{H}_{\| \mathrm{l}}$, the lamellar phases, and tubes) coexist in the system. Such lipid tubes are usually fabricated by pulling a part of vesicles or cell membranes with a pipet ${ }^{18-20}$ or optical tweezers manually. ${ }^{17}$ The present work shows that the lipid tubes form also from $\mathrm{H}_{\|}$without explicitly applying a point load manually.

Next, we discuss the physics behind the self-assembly. On the basis of the previous theoretical study on $\mathrm{DOPE}^{40}$ the total free energy of a number of lipids $\left(N=2 A / a\right.$, where $a \approx 0.65 \mathrm{~nm}^{2}$, from ref 40 , is the area per lipid molecule) corresponding to a bilayer of area $A$ can be expressed as

$$
\begin{gathered}
E_{\mathrm{H}}=\varepsilon_{\mathrm{i}} A \\
E_{\mathrm{L}}=\left\{\frac{\kappa}{2}\left(\frac{1}{R_{0}}\right)^{2}-W\right\} A \\
E_{\mathrm{T}}=\frac{\kappa}{2}\left[\frac{1}{2}\left(\frac{1}{R}-\frac{1}{R_{0}}\right)^{2}+\frac{1}{2}\left(-\frac{1}{R}-\frac{1}{R_{0}}\right)^{2}\right] A \\
=\frac{\kappa}{2}\left[\left(\frac{1}{R}\right)^{2}+\left(\frac{1}{R_{0}}\right)^{2}\right] A
\end{gathered}
$$

for the $\mathrm{H}_{\|}$phase, the lamellar phase, and a tube of radius $R(A=2 \pi R L$, where $L$ is the length of the tube), respectively. Because the $H_{\|}$phase is fully hydrated, the lipid monolayers assume their radius of spontaneous curvature $\left(R_{0} \approx 2.85 \mathrm{~nm}\right.$ from eq 12 in ref 40 and Table 1 in ref 41 ), so the free energy of the $H_{\|}$ phase $\left(E_{\mathrm{H}}\right)$ contains no elastic term and consists only of the interstitial energy (with energy density $\varepsilon_{\mathrm{i}}=2 g_{\mathrm{i}} /$ $a \approx 4.5 \mathrm{pN} / \mathrm{nm}$ from Table 1 in ref 40 , where $g_{\mathrm{i}}$ is the interstitial energy per lipid molecule), which originates from the voids in the hexagonal interstices (note that in ref 40 this term was included in $E_{\mathrm{L}}$ with a negative sign (-) since it is a difference between $E_{\mathrm{H}}$ and $E_{\mathrm{L}}$ ). In the free energy of the lamellar phase $\left(E_{\mathrm{L}}\right)$ the first term describes the elastic energy of the bilayers deformed to a flat configuration (characterized by the bending modulus $\kappa=2 \kappa_{\text {monolayer }} \approx$ $22 k T \approx 90 \mathrm{pNnm}$ ), ${ }^{41,42}$ while the second term (with energy density $-W$, which is of the order of $-0.1 \mathrm{pN} / \mathrm{nm}$ from the sum of the last two values of the first line 

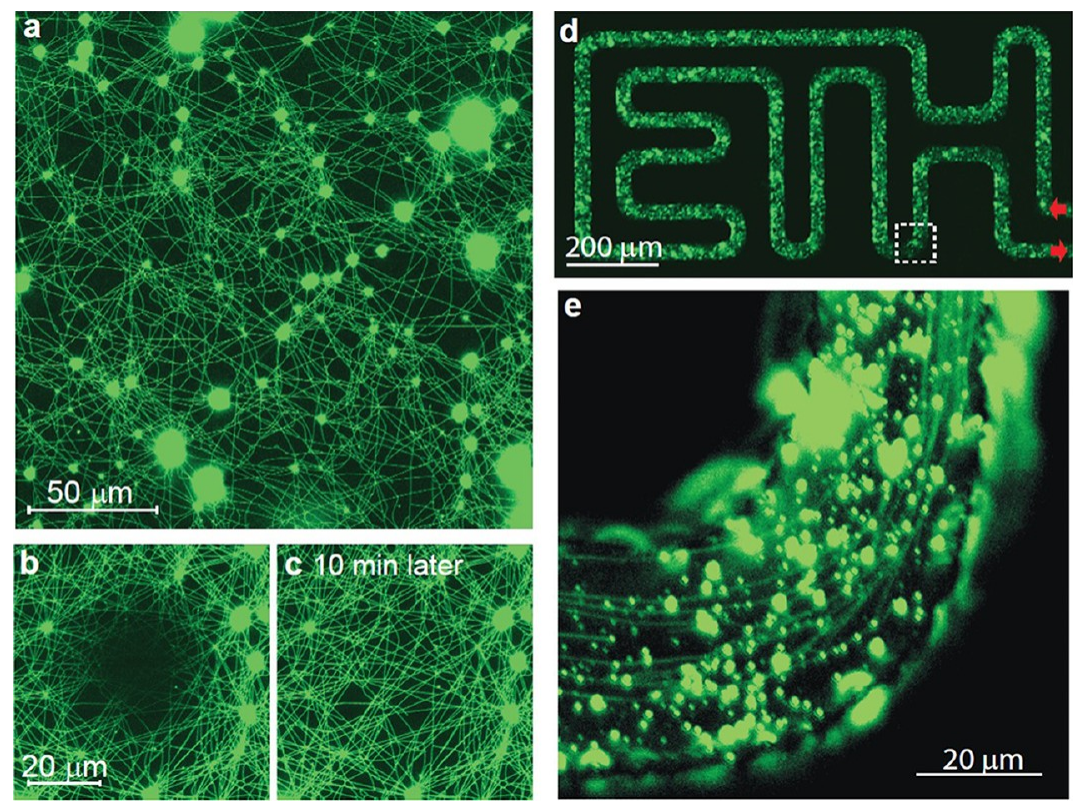

Figure 3. Lipid nanotube patterning. (a) A lipid nanotube network directed by a turbulent flow. Fluorescent recovery after photobleaching (FRAP) was carried out and an image (b) right after photobleaching and (c) 10 min later are shown. (d) "ETH" written with lipid nanotubes using a microfluidic system. The direction of inlet and outlet is indicated by arrows. (e) A zoomed-in image of a corner of " $\mathrm{H}$ " indicated with a white dotted square in panel $\mathrm{d}$, where the nanotubes curve along the channel.

of Table 2 in ref 40) comprises all the interactions between the bilayers (including Van der Waal attraction and hydration repulsion). Comparing $E_{\mathrm{H}}$ and $E_{\mathrm{L}}$ (with the above parameters) one can see that $E_{\mathrm{H}}<E_{\mathrm{L}}$, confirming that at room temperature the $H_{\|}$phase is more stable than the lamellar one. It is also in agreement with our croTEM images where the main core of the lipid blocks is in $\mathrm{H}_{\|}$phase.

The free energy of a bilayer tube $\left(E_{\mathrm{T}}\right)$, however, consists only of the usual elastic term (corresponding to two monolayer of curvatures $1 / R$ and $-1 / R$ ). Although the tubes are formed from the outer bilayer envelope of the blocks, their lipids are ultimately supplied by the $\mathrm{H}_{\|}$core. Therefore, the energy required to form a tube of radius $R$ and length $L$ is

$$
E(R)=E_{\mathrm{T}}(R)-E_{\mathrm{H}}(R)=\left(\frac{\kappa}{2 R^{2}}+\frac{\kappa}{2 R_{0}^{2}}-\varepsilon_{i}\right) 2 \pi R L
$$

In the case of stable tubes $E(R)$ takes its minimum with respect to $R$, leading to

$$
\frac{\kappa}{2 R_{\mathrm{opt}}^{2}}=\frac{\kappa}{2 R_{0}^{2}}-\varepsilon_{\mathrm{i}}
$$

from which the stable tube radius $\left(R_{\text {opt }}\right)$ can be expressed as

$$
R_{\mathrm{opt}}=\frac{R_{0}}{\sqrt{1-2 R_{0}^{2} \varepsilon_{i} / \kappa}} \approx 6.6 \mathrm{~nm}
$$

which is in remarkable agreement with the cryoTEM images (where $R_{\mathrm{opt}}$ is measured from the tube axis to the middle surface of the lipid tails as shown in the inset of Figure 2b), and explains why the optimal diameter of the tubes is independent of the applied flow.

The force required to form a tube can also be calculated as

$$
F=\frac{E\left(R_{\mathrm{opt}}\right)}{L}=\frac{2 \pi \kappa}{R_{\mathrm{opt}}}=86 \mathrm{pN}
$$

which must be provided by the solution flow. The drag force applied to a lipid block can be estimated as $F_{\text {Stokes }}=6 \pi \mu R_{\text {block }} V=3.8-380 \mathrm{pN}$, with the viscosity of water being $\mu=1 \times 10^{-3} \mathrm{~kg} / \mathrm{s} \cdot \mathrm{m}$ and the radius of the lipid block being $R_{\text {block }}=1-100 \mu \mathrm{m}$. For $v$, the velocity of the lipid block $v \approx 200 \mu \mathrm{m} / \mathrm{s}$ from Figure $1 \mathrm{~g}$ was used. This is a lower estimate for the initial flow velocity applied to the static block attached to the surface, yielding a lower estimate of the viscous forces exerted on the lipid blocks. This implies that the drag force is large enough to protrude a tube for large lipid blocks. The formation of the narrow neck between two lipid blocks at the beginning of the tube assembly may happen because of the point contact between a surface-bound and a floating block upon an impact. Indeed, the tube assembly occurs mostly when a flow is induced in the presence of lipid blocks in solution.

We will now present two examples of solution-flowdriven lipid-nanotube patterning to highlight its versatility. The first example is a lipid nanotube network that assembled directed by a turbulent flow. When a turbulent flow was applied by a pipet after the adsorption of the lipid blocks, lipid nanotubes formed a 2D network (Figure 3a). They were stable at least for 3 days 
in solution without changing their optical properties. The addition of solution with different salt concentrations (the osmolarity difference of more than $100 \mathrm{mM}$ ) changes their optical appearance (tubes widen and dots appear on them), indicating that the structure is relatively sensitive to the osmotic pressure difference between the in- and outside of the tubes. The lipid nanotubes have an internal molecular mobility, with an estimated diffusion coefficient of $D \approx 6 \times 10^{-9} \mathrm{~cm}^{2} / \mathrm{s}$ based on fluorescent recovery after photobleaching (FRAP) with a 2D FRAP model (Figure $3 b, c) .{ }^{43}$ Such an estimation of the diffusion coefficient of the $1 D$ lipid structure was possible because the nanotubes were surface-bound. The values are of the same order of magnitude as those for lipid bilayers on glass surfaces. ${ }^{44}$ This lipid nanotube network could be used as a scaffold for cells similarly to nanofibers formed by self-assembly of peptide amphiphile molecules that allowed the selective differentiation of neural progenitor cells encapsulated in the network. ${ }^{45}$ The second example is writing with lipid nanotubes. A microfluidic flow-cell with an ETHshaped channel (width and height of $50 \mu \mathrm{m}$ ) was fabricated with PDMS (Figure 3d). To establish only lamellar flow without any turbulence, particular care was taken to design the channel without sharp edges. The high magnification image of the curved edge (Figure 3e) shows that the lipid nanowires were able to curve along the channel, while FRAP confirmed their internal mobility.

\section{CONCLUSION}

The reported system is distinct from other reported tube fabrications because (1) it involves three lipid phases, (i.e., $\mathrm{H}_{\|}$, the lamellar phases, and tubes) while the others include only lamellae and tubes, and (2) the lipid nanotubes form without explicitly applying a point load. The two points are mostly interconnected, because the loose lamellar envelope supported by the inner $\mathrm{H}_{\|}$structure may enable the point contact to the surface or to another lipid block just upon a fluctuation of solution. On the other hand, the surface of the vesicles is so smooth that the point load has to be applied manually. The capability of freely aligning lipid nanotubes on surfaces in a highthroughput manner could be used for molecular patterning by attaching molecules on the tubes. The nanotubes have inner fluidity, which may allow for further electrical/magnetic/fluid-dynamical manipulation of lipids or lipid-attached molecules. In addition, the tube structure with a uniform diameter could be used for nanocapsule and nanowire fabrication by metallization, all of which can be patterned on surfaces. From a biological perspective, the finding that a lipid abundant in bacteria self-assembles into nanotubes, transports molecules along the nanotubes, and disappears by itself under a physiological condition directed by a solution flow may provide new explanations to poorly understood biological processes, such as the molecular transport between eukaryotic cells ${ }^{15,17}$ or between bacteria. ${ }^{16}$

\section{MATERIALS AND METHODS}

Buffer Solution (HEPES). All the experiments were performed in a buffer solution at $\mathrm{pH}$ 7.4. The buffer solution (HEPES) was prepared with $10 \mathrm{mM} \mathrm{4-(2-hydroxyethyl)piperazine-1-ethane-}$ sulfonic acid purchased from Fluka (Buchs, Switzerland) and $0.15 \mathrm{M}$ sodium chloride (Fluka, Buchs, Switzerland) in ultrapure water filtered through Milli-Q Gradient A10 filters (Millipore AG, Switzerland). The $\mathrm{pH}$ was adjusted to 7.4 using $6 \mathrm{M} \mathrm{NaOH}$ (Fluka, Buchs, Switzerland).

Polyelectrolytes. Polyethyleneimine (PEI, MW $=25000 \mathrm{~g} / \mathrm{mol}$, branched, 408727 ) and poly-L-lysine hydrobromide (PLL, MW = 15000 to $30000 \mathrm{~g} / \mathrm{mol}$, P7890) were purchased from Sigma-Aldrich Chemie GmbH (Switzerland). PEI and PLL were dissolved at a concentration of $1 \mathrm{mg} / \mathrm{mL}$ in HEPES. All the solutions were sterile filtrated through $0.22 \mu \mathrm{m}$ filters.

DOPE Lipid Solutions. 1,2-Dioleoyl-sn-glycero-3-phosphoethanolamine (DOPE) and 1-oleoyl-2-[12-[(7-nitro-2-1,3-benzoxadiazol-4-yl)amino]lauroyl]-sn-glycero-3-phosphocholine (NBDPC) were purchased from Avanti Polar Lipids and stored in chloroform. For both the optical imaging and the cryoSEM imaging, the lipid solution was prepared by taking DOPE + $2 \%$ NBD-PC into a flask, drying and adding a physiological buffer solution, followed by a sonication at the highest power using Elma Transsonic Digital S (T710DH, IS@Work Pte Ltd., Singapore) at a final concentration of $1 \mathrm{mg} / \mathrm{mL}$. Lipid nanotubes formed also with $100 \%$ DOPE lipids, confirmed by a fluorescent microscope by encapsulating Calcein dissolved buffer solution inside of the nonlabeled tubes. Except for the NBD-PC used in the manuscript, we also use other fluorophore-tagged lipids such as BODIPY FL HPA (invitrogen) and Liss Rhodamine-PE (Avanti Lipids), and they all show similar nanotube assemblies. Those three fluorophore-tagged lipids have differences in terms of the shape (headgroup-tagged or tail-group-tagged, thus conical or inverse conical) and electrical charge. It suggests that the addition of different lipids at this concentration does not affect the formation of the tubes significantly.

Flowcells. Straight flowcells consist of a sticky-Slide I 0.4 Luer (ibidi, Germany), and a glass slide cleaned by an oxygen-plasma cleaner (PDC-32G, Harrick, USA) just before the experiments. The channel was $50 \mathrm{~mm}$ long, $5 \mathrm{~mm}$ wide, and $0.4 \mathrm{~mm}$ high. The PDMS flowcell with an ETH-shaped channel was fabricated by replica molding. ${ }^{46,47}$ Briefly, the channel pattern was fabricated on a Si wafer by photolithography with SU-8 photoresist spin-coated at a thickness of $\sim 50 \mu \mathrm{m}$. A precursor mixture of poly(dimethylsiloxane), PDMS, was cast and cured on the Si wafer. The device could then be peeled off from the Si master after baking.

Confocal Laser Scanning Microscope (CLSM) and Fluorescent Recovery after Photobleaching (FRAP). We used a CLSM 510 microscope (Zeiss, Germany) equipped with an argon laser (488 $\mathrm{nm}$ ) using a $40 \times($ LD, NA 0.7$)$ objective. The FRAP data were analyzed by evaluating the intensity with ImageJ (Image processing and analysis in Java, National Institute of Health; http://rsb.info.nih. gov). The diffusion coefficient was determined with the fitting procedure described in ref 43.

CCD Camera Used for Movie. Movies, from which snapshots were taken for figures, were recorded by the microscope and 
a CCD camera (C9100-13) purchased from Hamamatsu, Japan. The contrast between the nanowires and the background was enhanced by Histogram Equalized with the software provided by the same company.

Cryotransmission Electron Microscopy (Cryo-TEM). For the cryo-TEM imaging, the samples were fabricated on PEl-coated glass slides in the same way with DOPE $+2 \%$ NBD-PC. Subsequently, the samples were transferred onto glow-discharged holey carboncoated grid (quantifoil, Germany) by touching the sample surface with the grid, immediately blotted with Whatman filter paper and vitrified into liquid ethane at $-178^{\circ} \mathrm{C}$ using a vitrobot (FEl company, Netherlands). Frozen grids were transferred onto a Philips CM200-FEG electron microscope using a Gatan 626 cryo-holder (GATAN, USA). Electron micrographs were recorded at an accelerating voltage of $200 \mathrm{kV}$ and a nominal magnification of $50000 \times$, using a low-dose system $\left(10 \mathrm{e}^{-} / \AA^{2}\right)$ and keeping the sample at $-175^{\circ} \mathrm{C}$. Defocus values were $-2.5 \mu \mathrm{m}$. Micrographs were recorded at $4 \mathrm{~K} \times 4 \mathrm{~K}$ CMOS camera (TVIPS, Germany). The diameter of the lipid nanotube was estimated by measuring the diameter at different places on tubes (totally 90 data points from six different tubes).

Conflict of Interest: The authors declare no competing financial interest.

Acknowledgment. This work was supported by EU Seventh Research Framework Program (FP7, ASMENA Project). We are grateful to Stephen Wheeler (LBB workshop), Martin Lanz (LBB workshop), and Michael Gabi (LBB) for the fabrication of the microfluidic cells, and to Prof. Henning Stahlberg (Director of C-CINA, Biozentrum, University of Basel) for his support and access to the electron microscopy facilities. The electron microscopy part is supported by the Swiss National Science Foundation (SystemsX.ch RTD CINA)

Supporting Information Available: (Figure S1) snapshots from a movie recording the lipid nanotube formation; (Figure S2) confocal laser scanning microscope (CLSM) images of DOPE lipids adsorbed on a poly-L-lysine (PLL)-coated glass slide and on a bare glass slide; (Movie S1) a movie taken with a fluorescent optical microscope showing the directed self-assembly of lipid nanotubes. This material is available free of charge via the Internet at http://pubs.acs.org

\section{REFERENCES AND NOTES}

1. Schnur, J. M. Lipid Tubules: A Paradigm for Molecularly Engineered Structures. Science 1993, 262, 1669-1676.

2. Nakashima, N.; Asakuma, S.; Kunitake, T. Optical Microscopic Study of Helical Superstructures of Chiral Bilayer Membranes. J. Am. Chem. Soc. 1985, 107, 509-510.

3. Yamada, K.; Ihara, H.; Ide, T.; Fukumoto, T.; Hirayama, C. Formation of Helical Super Structure from Single-Walled Bilayers by Amphiphiles with Oligo-L-Glutamic Acid-Head Group. Chem. Lett. 1984, 1713-1716.

4. Yager, P.; Schoen, P. E. Formation of Tubules by a Polymerizable Surfactant. Mol. Cryst. Liq. Cryst. 1984, 106, 371-381.

5. Papahadjopoulos, D.; Jacobson, K.; Nir, S.; Isac, I. Phase Transitions in Phospholipid Vesicles Fluorescence Polarization and Permeability Measurements Concerning the Effect of Temperature and Cholesterol. Biochim. Biophys. Acta, Biomembr. 1973, 311, 330-348.

6. Tocanne, J. F.; Ververgaert, P. H. J. T.; Verkleij, A. J.; van Deenen, L. L. M. A Monolayer and Freeze-Etching Study of Charged Phospholipids I. Effects of lons and Ph on the Ionic Properties of Phosphatidylglycerol and Lysylphosphatidylglycerol. Chem. Phys. Lipids 1974, 12, 201-219.

7. Chappell, J. S.; Yager, P. Formation of Mineral Microstructures with a High Aspect Ratio from Phospholipid Bilayer Tubules. J. Mater. Sci. Lett. 1992, 11, 633-636.

8. Archibald, D. D.; Mann, S. Structural Studies of Lipid Fibers Formed by Sphingosine. Biochim. Biophys. Acta, Lipids Lipid Metab. 1993, 1166, 154-162.

9. Huetz, P.; van Neuren, S.; Ringler, P.; Kremer, F.; van Breemen, J. F. L.; Wagenaar, A.; Engberts, J. B. F. N.; Fraaije, J. G. E. M.; Brisson, A. Relationship between Molecular Structure and Supramolecular Morphology of Doda-Eo2-Biotin and Related Lipids. Chem. Phys. Lipids 1997, 89, 15-30.

10. Rosenblatt, C.; Yager, P.; Schoen, P. E. Orientation of Lipid Tubules by a Magnetic-Field. Biophys. J. 1987, 52, 295-301.

11. Hayes, M. A.; Pysher, M. D.; Chen, K. P. Liposomes Form Nanotubules and Long Range Networks in the Presence of Electric Field. J. Nanosci. Nanotechnol. 2007, 7, 2283-2286.

12. Mahajan, N.; Fang, J. Two-Dimensional Ordered Arrays of Aligned Lipid Tubules on Substrates with Microfluidic Networks. Langmuir 2005, 21, 3153-3157.

13. Frusawa, H.; Fukagawa, A.; Ikeda, Y.; Araki, J.; Ito, K.; John, G.; Shimizu, T. Aligning a Single-Lipid Nanotube with Moderate Stiffness. Angew. Chem., Int. Ed. 2003, 42, 72-74.

14. Zhu, T. F.; Szostak, J. W. Growth, Coupled, and Division of Model Protocell Membranes. J. Am. Chem. Soc. 2009, 131, 5705-5713.

15. Rustom, A.; Saffrich, R.; Markovic, I.; Walther, P.; Gerdes, H. H. Nanotubular Highways for Intercellular Organelle Transport. Science 2004, 303, 1007-1010.

16. Dubey, G. P.; Ben-Yehuda, S. Intercellular Nanotubes Mediate Bacterial Communication. Cell 2011, 144, 590-600.

17. Pascoal, P.; Kosanic, D.; Gjoni, M.; Vogel, H. Membrane Nanotubes Drawn by Optical Tweezers Transmit Electrical Signals between Mammalian Cells over Long Distances. Lab Chip 2010, 10, 2235-2241.

18. Karlsson, A.; Karlsson, R.; Karlsson, M.; Cans, A.-S.; Stromberg, A.; Ryttsen, F.; Orwar, O. Molecular Engineering: Networks of Nanotubes and Containers. Nature 2001, 409, 150-152.

19. Heinrich, V.; Bozic, B.; Svetina, S.; Zeks, B. Vesicle Deformation by an Axial Load: From Elongated Shapes to Tethered Vesicles. Biophys. J. 1999, 76, 2056-2071.

20. Evans, E.; Bowman, H.; Leung, A.; Needham, D.; Tirrell, D. Biomembrane Templates for Nanoscale Conduits and Networks. Science 1996, 273, 933-935.

21. Rossier, O.; Cuvelier, D.; Borghi, N.; Puech, P. H.; Derényi, I.; Buguin, A.; Nassoy, P.; Brochard-Wyart, F. Giant Vesicles under Flows: Extrusion and Retraction of Tubes. Langmuir 2003, 19, 575-584.

22. Heinrich, V.; Leung, A.; Evans, E. Nano- to Microscale Dynamics of P-Selectin Detachment from Leukocyte Interfaces. II. Tether Flow Terminated by P-Selectin Dissociation from PSGL-1. Biophys. J. 2005, 88, 2299-2308.

23. Borghi, N.; Brochard-Wyart, F. Tether Extrusion from Red Blood Cells: Integral Proteins Unbinding from Cytoskeleton. Biophys. J. 2007, 93, 1369-1379.

24. Dittrich, P. S.; Heule, M.; Renaud, P.; Manz, A. On-Chip Extrusion of Lipid Vesicles and Tubes through Microsized Apertures. Lab Chip 2006, 6, 488-493.

25. Lin, Y.-C; Huang, K.-S; Chiang, J.-T; Yang, C.-H.; Lai, T.-H. Manipulating Self-Assembled Phospholipid Microtubes Using Microfluidic Technology. Sens. Actuators, B 2006, 117, 464-471.

26. West, J.; Manz, A.; Dittrich, P. S. Lipid Nanotubule Fabrication by Microfluidic Tweezing. Langmuir 2008, 24, 6754-6758.

27. Tan, Y.-C.; Shen, A. Q.; Li, Y.; Elson, E.; Ma, L. Engineering Lipid Tubules Using Nano-Sized Building Blocks: The Combinatorial Self-Assembly of Vesicles. Lab Chip 2008, 8, 339-345.

28. Koynova, R.; Caffrey, M. Phases and Phase Transitions of the Hydrated Phosphatidylethanolamines. Chem. Phys. Lipids 1994, 69, 1-34

29. Rand, R. P.; Fuller, N. L. Structural Dimensions and Their Changes in a Reentrant Hexagonal-Lamellar Transition of Phospholipids. Biophys. J. 1994, 66, 2127-2138.

30. Shalaev, E. Y.; Steponkus, P. L. Phase Diagram of 1,2-Dioleoylphosphatidylethanolamine (Dope):Water System at Subzero Temperatures and at Low Water Contents. Biochim. Biophys. Acta, Biomembr. 1999, 1419, 229-247.

31. Siegel, D. P.; Green, W. J.; Talmon, Y. The Mechanism of Lamellar-to-Inverted Hexagonal Phase Transitions: A Study Using Temperature-Jump Cryo-electron Microscopy. Biophys. J. 1994, 66, 402-414.

32. Van Venetië, R.; Verkleij, A. J. Analysis of the Hexagonal II Phase and Its Relations to Lipidic Particles and the 
Lamellar Phase a Freeze-Fracture Study. Biochim. Biophys. Acta, Biomembr. 1981, 645, 262-269.

33. Ishida, Y.; Kitagawa, K.; Nakayama, A.; Ohtani, H. Complementary Analysis of Lipids in Whole Bacteria Cells by Thermally Assisted Hydrolysis and Methylation-GC and MALDI-MS Combined with on-Probe Sample Pretreatment. J. Anal. Appl. Pyrol. 2006, 77, 116-120.

34. Kamal, M. M.; Mills, D.; Grzybek, M.; Howard, J. Measurement of the Membrane Curvature Preference of Phospholipids Reveals Only Weak Coupling between Lipid Shape and Leaflet Curvature. Proc. Natl. Acad. Sci. 2009, 106, 22245-22250.

35. Churchward, M. A.; Rogasevskaia, T.; Brandman, D. M.; Khosravani, H.; Nava, P.; Atkinson, J. K.; Coorssen, J. R. Specific Lipids Supply Critical Negative Spontaneous Curvature-An Essential Component of Native $\mathrm{Ca}^{2+}$-Triggered Membrane Fusion. Biophys. J. 2008, 94, 3976-3986.

36. Moya, S. E.; Toca-Herrera, J. L. From Hollow Shells to Artificial Cells: Biointerface Engineering on Polyelectrolyte Capsules. J. Nanosci. Nanotechnol. 2006, 6, 2329-2337.

37. Siegel, D. P.; Epand, R. M. The Mechanism of Lamellarto-Inverted Hexagonal Phase Transitions in Phosphatidylethanolamine: Implications for Membrane Fusion Mechanisms. Biophys. J. 1997, 73, 3089-3111.

38. Barauskas, J.; Johnsson, M.; Tiberg, F. Self-Assembled Lipid Superstructures: Beyond Vesicles and Liposomes. Nano Lett. 2005, 5, 1615-1619.

39. Schwarz, G.; Callewaert, G.; Droogmans, G.; Nilius, B. Shear Stress-Induced Calcium Transients in Endothelial-Cells from Human Umbilical-Cord Veins. J. Physiol., London 1992, 458, 527-538.

40. Kozlov, M. M.; Leikin, S.; Rand, R. P. Bending, Hydration and Interstitial Energies Quantitatively Account for the Hexagonal-Lamellar-Hexagonal Reentrant Phase-Transition in Dioleoylphosphatidylethanolamine. Biophys. J. 1994, 67, 1603-1611.

41. Chen, Z.; Rand, R. P. The Influence of Cholesterol on Phospholipid Membrane Curvature and Bending Elasticity. Biophys. J. 1997, 73, 267-276.

42. Leikin, S.; Kozlov, M. M.; Fuller, N. L.; Rand, R. P. Measured Effects of Diacylglycerol on Structural and Elastic Properties of Phospholipid Membranes. Biophys. J. 1996, 71, 2623-2632.

43. Soumpasis, D. M. Theoretical Analysis of Fluorescence Photobleaching Recovery Experiments. Biophys. J. 1983, 41, 95-97.

44. Stelzle, M.; Miehlich, R.; Sackmann, E. Two-Dimensional Microelectrophoresis in Supported Lipid Bilayers. Biophys. J. 1992, 63, 1346-1354.

45. Silva, G. A.; Czeisler, C.; Niece, K. L.; Beniash, E.; Harrington, D. A.; Kessler, J. A.; Stupp, S. I. Selective Differentiation of Neural Progenitor Cells by High-Epitope Density Nanofibers. Science 2004, 303, 1352-1355.

46. Dusseiller, M. R.; Niederberger, B.; Stadler, B.; Falconnet, D.; Textor, M.; Voros, J.; Novel Crossed, A Microfluidic Device for the Precise Positioning of Proteins and Vesicles. Lab Chip 2005, 5, 1387-1392.

47. Xia, Y.; Whitesides, G. M. Soft Lithography. Angew. Chem., Int. Ed. 1998, 37, 550-575. 